\title{
Photometry and Multipolar Magnetic Field Modeling of Polars: BY Camelopardalis and FL Ceti
}

\author{
P. A. Mason ${ }^{1,2}$, A. G. Zhilkin ${ }^{3}$, D. V. Bisikalo ${ }^{3}$, S. Gomez ${ }^{1}$, J. Morales ${ }^{1}$, E. L. Robinson ${ }^{4}$ \\ ${ }^{1}$ Department of Physics, University of Texas at El Paso, El Paso TX, USA \\ ${ }^{2}$ Department of Mathematics and Physical Sciences, New Mexico State University - DACC, Las Cruces NM, USA \\ ${ }^{3}$ Institute of Astronomy, Russian Academy of Sciences, Moscow, Russia \\ ${ }^{4}$ Department of Astronomy, University of Texas at Austin, Austin TX, USA
}

Corresponding author: pmason@nmsu.edu

\begin{abstract}
We present new broad band optical photometry of two magnetic cataclysmic variable stars, the asynchronous polar BY Camelopardalis and the short period polar FL Ceti. Observations were obtained at the 2.1-m Otto Struve Telescope of McDonald Observatory with $3 \mathrm{~s}$ and 1s integration times respectively. In an attempt to understand the observed complex changes in accretion flow geometry observed in BY Cam, we performed full 3D MHD simulations assuming a variety of white dwarf magnetic field structures. We investigate fields with increasing complexity including both aligned and non-aligned dipole plus quadrupole field components. We compare model predictions with photometry at various phases of the beat cycle and find that synthetic light curves derived from a multipolar field structure are broadly consistent with optical photometry. FL Ceti is observed to have two very small accretion regions at the foot-points of the white dwarf's magnetic field. Both accretion regions are visible at the same time in the high state and are about 100 degrees apart. MHD modeling using a dipole plus quadrupole field structure yields quite similar accretion regions as those observed in FL Ceti. We conclude that accretion flows calculated from MHD modeling of multi-polar magnetic fields produce synthetic light curves consistent with photometry of these magnetic cataclysmic variables.
\end{abstract}

Keywords: cataclysmic variables - magnetic fields - polars - optical - photometry - individual: BY Cam - individual: FL Cet.

\section{Introduction}

We report on new observations of magnetic cataclysmic variable stars (mCVs). Photometric observations of two mCVs: BY Cam and FL Ceti, were obtained at McDonald Observatory using the Otto Struve 2.1-m telescope. The data consists of 1 or 3 second exposures and no dead time. A broad band filter covering the visual spectrum, BVR, was used in order to obtain the highest possible time resolution. We describe progress towards understanding the structure of accretion flows in $\mathrm{mCVs}$, obtained through the use of full 3D MHD simulations assuming a variety of magnetic field structures.

\section{BY Camelopardalis}

BY Cam contains a highly magnetic white dwarf which rotates slightly (about $1 \%$ ) faster than its companion (donor star) orbits (Mason et al. 1989, 1998; Silber et al. 1992, 1997). Hence, it is a member of the class of mCVs known as asynchronous polars or BY Cam stars.

The binary period is 201.3 minutes while the white dwarf spin period is 199.3 minutes (Mason et al. 1995).
A precise orbital ephemeris was derived by Schwarz et al. (2005). The vast majority of polars are synchronously locked due to the strong magnetic field of the white dwarf primary. Despite the fact that BY Cam has a magnetic field typical of polars, it currently is in an unlocked state. Mason and Chanmugam (1992) and Pavlenko et al. (2012) provide evidence for the synchronization of the orbit and spin periods in 1600 and 250 years respectively.

BY Cam is well suited for the study of magnetic field structure in mCVs because we are able to take advantage of asynchronous rotation of the white dwarf with respect to the binary. Synchronized polars, by definition, only accrete from one azimuthal angle. In BY Cam, accretion flow occurs at all azimuthal angles as the field structure moves with respect to the orbital plane over the course of a 14 day beat cycle (Mason et al. 1995, 1998), see Figure 1. Other extensive photometric studies of BY Cam were conducted by Andronov et al. (2008) and Babina et al. (2010). 

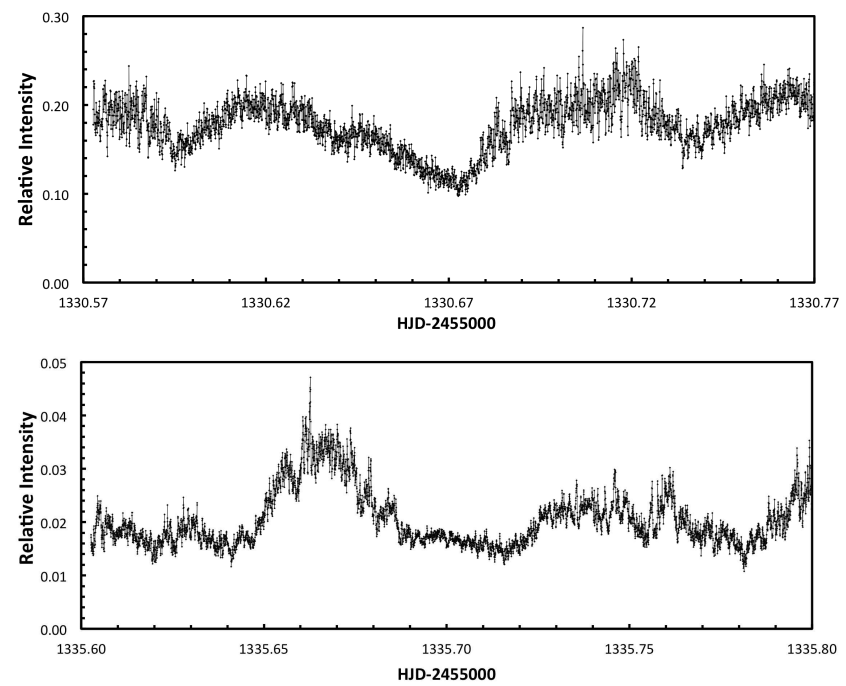

Figure 1: BY Cam photometry. Top: B-band from 7 February 2013. Bottom: R-band from 12 February 2013. Cycle to cycle variations are clearly evident.

\subsection{BY Cam: MHD modeling}

A complex magnetic field model was proposed to explain optical photometry and polarimetry of BY Cam by Mason et al. (1998). Full 3D MHD simulations of flow structure in asynchronous polars were carried out using the Russian supercomputer facility (Zhilkin et al. 2012).

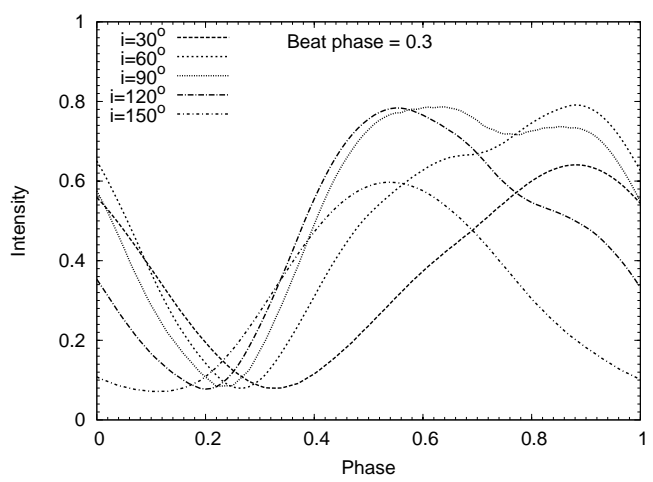

Figure 2: Synthetic light curves derived from a nonaligned dipole plus quadrupole field model at beat phase 0.3 are shown as an example. These light curves resemble some of the observed light curves of BY Cam, see Figure 1.

Calculations of accretion flow patterns at 10 beat phases were performed. for pure dipole and dipole plus quadrupole field components of various dipole/quadrupole field strength ratios. Figures 2 and 3 show synthetic light curves of a BY Cam model at two beat phases.

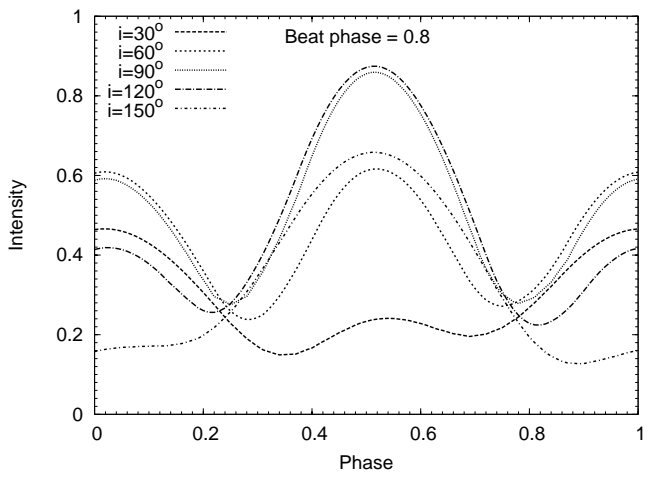

Figure 3: Same as Figure 2, except for beat phase 0.8. These light curves resemble some of the observed light curves of BY Cam, see Figure 1.

Figure 4 shows the magnetic induction at the surface of the white dwarf for a misaligned dipole plus quadrupole magnetic field model, currently the best fitting model for BY Cam. Figure 5 shows the location of accretion regions for an aligned dipole plus quadrupole field structure at a particular beat phase.

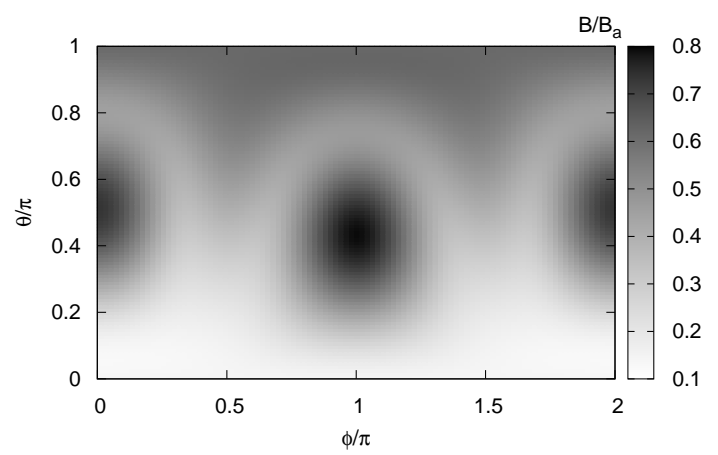

Figure 4: The magnetic induction over the surface of the model white dwarf in BY Cam. Dark areas represent locations of potential accretion. There are three regions, two near the magnetic equator and one at the north magnetic pole. This is a non-aligned dipole plus quadrupole field model.
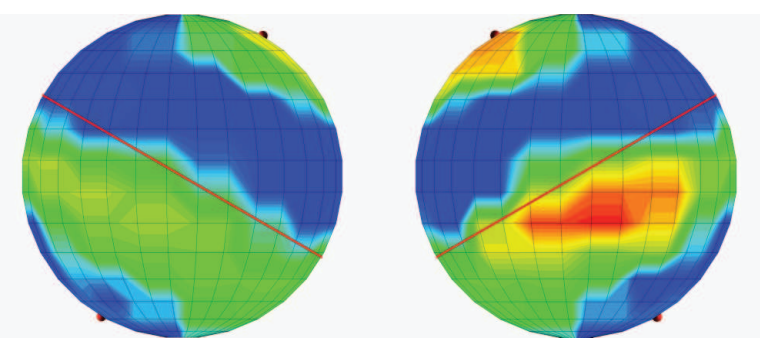

Figure 5: An example model of BY Cam with dipole plus quadrupole components with accretion regions, just below the magnetic equator (see right panel) and the magnetic north pole. 
Photometry and Multipolar Magnetic Field Modeling of Polars: BY Camelopardalis and FL Ceti

\section{FL Ceti}

The original optical observations of this synchronized Polar revealed deep eclipses on a period of $87 \mathrm{~min}$ (Szkody et al. 2002). It has been observed undergoing $\sim 6$ mag. eclipses in the high state, with $\sim 1.5$ mag. eclipses in the low state. It has been the subject of numerous additional investigations (Dubkova et al., 2003; Wiehahn et al. 2004, Woudt et al., 2004, Schmidt et al., 2005, O'Donoghue et al. 2006). Katysheva and Shugarov (2012) provide a concise review of past work and present additional data.

We obtained optical photometry of FL Ceti, during both high and low states, using the McDonald Observatory Otto Struve 2.1-m telescope. Fast photometry allows us to resolve detailed eclipse structure resulting from the very small accretion regions on the surface of the white dwarf. Eclipse's of the accretion spots have ingress and egress times of 2-3 seconds, indicating a very small structure. Polars are known for exhibiting high and low brightness states, corresponding to high and low accretion rates respectively. In some cases, low states may be periods of near zero accretion.

Broad-band (BVR) photometry was collected in $\mathrm{Au}-$ gust and September 2011 during which time FL Ceti was in the high state with two active accretion regions (see Figure 6). Low state light curves were obtained on six nights in October 2013. V-band photometry obtained 7 October 2013 is shown in Figure 7. The light is dominated by the white dwarf at a relatively constant brightness. The low amplitude variability in the out of eclipse light curve may be due to ellipsoidal variations of the secondary. That is, the visible surface area of the non-spherical donor star changes over the course of the orbital period. During the eclipse the back side of the secondary provides the only contribution to the light during both high and low states.

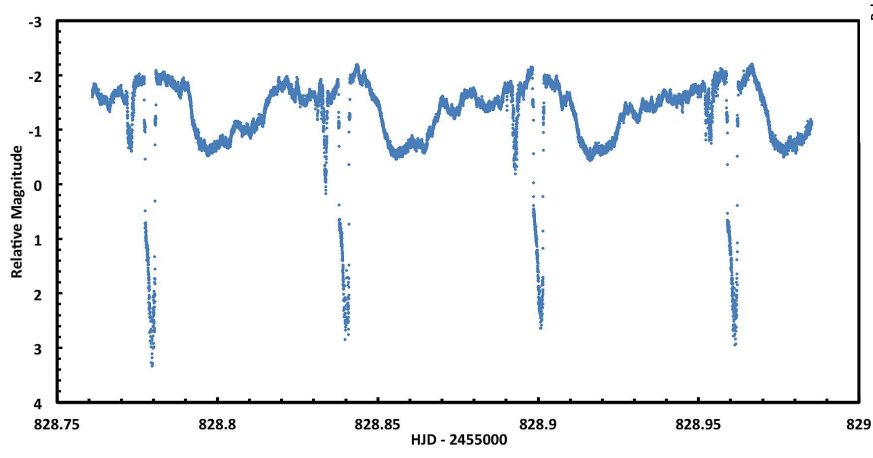

Figure 6: Broad-band (BVR) photometry obtained during a high accretion state of FL Ceti. Outside of eclipse the light curve is dominated by cyclotron emission. During the eclipse the back side of the secondary and the disappearance of the accretion stream provide the only contribution to the optical light.
In order to compare the high and low states a close up view of eclipses of the white dwarf by the secondary star are shown in Figure 8, for the high state (top) and the low state (bottom). The high state eclipse involves two sharp drops during ingress and a stand still in between. The egress is symmetric about mid-eclipse of the two spots. This is interpreted as the eclipse of two small accretion spots on the surface of the white dwarf (O'Donoghue, et al. 2006). One spot is eclipsed for a somewhat longer period of time than the other. Low state flux is flat during the deep eclipse while the high state light curve shows a gradual decline. An explanation for the gradual fading during eclipse has been given by O'Donoghue et al., (2006), that light from the accretion stream is being gradually blocked during the eclipse.

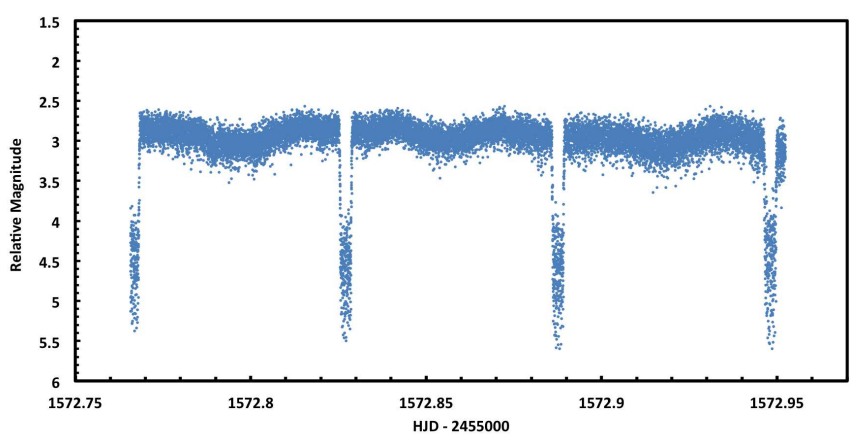

Figure 7: V-band photometry obtained during a low accretion state of FL Ceti.

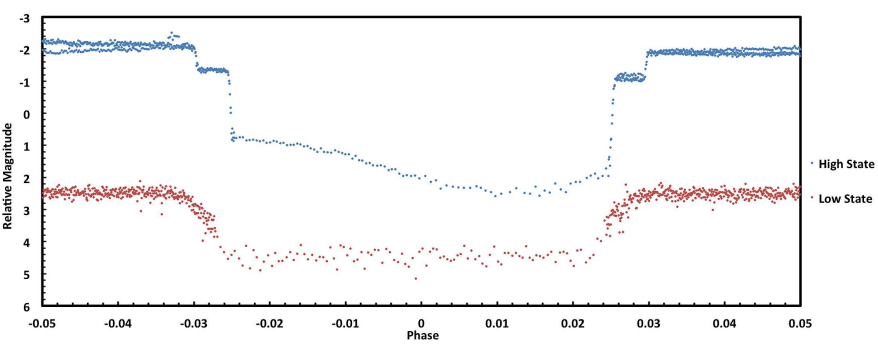

Figure 8: Eclipses of the white dwarf by the secondary star are shown for the high state (top - red points) and low state (bottom - blue points). The orbital phase is shown, with phase 0 being the time of mid-eclipse.

\subsection{FL Ceti: MHD modeling}

As described in the previous section, Zhilkin et al. (2012) performed calculations of MHD accretion flow in an asynchronous polar at 10 beat phases. The same calculations are used to model the field structure synchronous polars. Conveniently, FL Ceti has a measured magnetic field strength very similar to BY Cam. A first 
attempt at an accretion flow model resembling FL Ceti is shown in Figure 9. In this case, for MHD calculations, a strong quadrupole field component is assumed to be aligned with a dipole component. The donor star is at the right edge of the image and the stream phasing is similar to the pre eclipse dip observed in the light curve of FL Ceti. Plasma flows onto two small regions located about 100 degrees apart on the surface of the white dwarf. Similar features occur for calculations involving non-aligned dipole plus quadrupole magnetic field components.

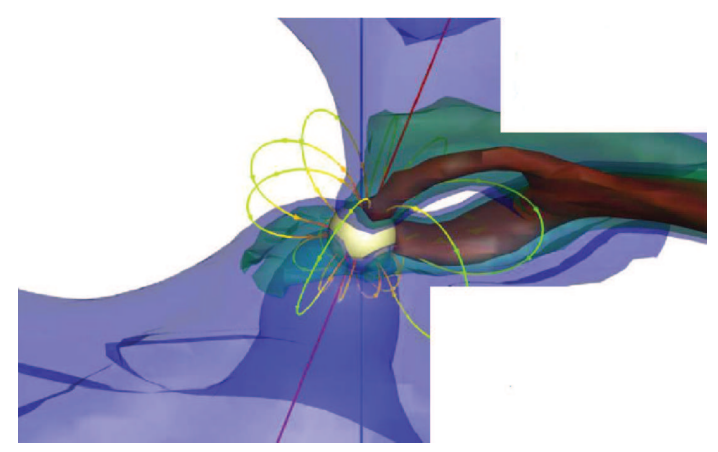

Figure 9: Model accretion flow in FL Ceti. The magnetic field structure is calculated for a centered dipole plus strong quadrupole, where the component axes are aligned. The donor star is off the far right edge on the horizontal axis. The densest part of the flow is shown in brown. Accretion stream phasing is consistent with the observed pattern in FL Ceti, specifically the pre-eclipse dip and the existence of magnetic poles separated by 100 degrees. In the model, one spot is at the magnetic north pole while the other is near the intersection of the magnetic equator and the orbital plane. Similar flow patterns result for misaligned dipole and quadrupole components as well.

\section{Conclusions}

It is clear that pure dipolar fields are inconsistent with observations of both BY Cam and FL Ceti. By modeling accretion flows in these binaries with higher order complex fields we are able to explain the evolution of the light curves of BY Cam and the location of accretion spots in FL Ceti. Actual fields may consist of many multipolar components. However, simple assumptions about complex field components yield results that are quite consistent with observations.

\section{Acknowledgement}

Special thanks go to Franco Giovannelli for the invitation to present this work in Palermo and to the ref- eree, Klaus Reinsch, for suggestions that improved the manuscript. We thank David Buckley for discussions concerning SALT data on FL Ceti. This work is supported, in part, by NSF/PAARE grant No. 0958783. AG and DB were supported by RFBR grants.

\section{References}

[1] Andronov, I. L., et al., 2008, CEJPh, 6, 385

[2] Babina, Ju., Andreev, M., Pavlenko, E., 2010, AIPC, 1273, 313

[3] Dubkova, D. N.; Kudryavtseva, N. A.; Hirv, A., 2003, IBVS, 5389, 1

[4] Katysheva, N., Shugarov, S. 2012, Memorie della Societa Astronomica Italiana, 83, 670

[5] Mason, P. A., Liebert, J., and Schmidt, G. D., 1989, ApJ, 346, 941

[6] Mason P. A. and Chanmugam G, 1992, ASPC, 29, 216

[7] Mason, P. A., et al., 1995, ASPC, 85, 496

[8] Mason, P. A., et al. 1998, MNRAS, 295, 511

[9] O'Donoghue, D., et al., 2006, MNRAS, 372, 151 doi:10.1111/j.1365-2966.2006.10834.x

[10] Pavlenko, E., et al, 2013, ASPC, 469, 343

[11] Schmidt, G. D., et al., 2005, ApJ, 620, 422 doi:10.1086/426807

[12] Schwarz, R., Schwope, A. D., Staude, A., Remillard, R. A., 2005, A \& A, 444, 213

[13] Silber, A. D., et al., 1992, ApJ 389, 704 doi:10.1086/171243

[14] Silber, A. D., et al., 1997, MNRAS, 290, 25 doi:10.1093/mnras/290.1.25

[15] Szkody, P. et al. 2002, AJ, 123, 430

[16] Wiehahn, M., Potter S. B., Warner, B., Woudt, P. A., 2004, MNRAS, 355, 689 doi:10.1111/j.1365-2966.2004.08346.x

[17] Woudt, P. A., Warner, B., Pretorius, M. L., 2004, MNRAS, 351, 1015 doi:10.1111/j.1365-2966.2004.07843.x

[18] Zhilkin A, et al.: 2012, Astron. Rep. 52, 4, 318 


\section{DISCUSSION}

PAULA SZKODY: How do the new light curves of BY Cam compare to those obtained in the past, by you and Silber et al.?
PAUL MASON: The new light curves resemble the light curves from 25 years ago. However, it should be noted that recently E. Pavlenko and co-workers suggest that BY Cam is in the process of synchronizing in about 250 years, a lot faster than the 1600 years estimated by Mason and Chanmugam (1992). 